\title{
Development and validation of risk and prognostic nomograms for bone metastases in Chinese advanced colorectal cancer patients
}

\author{
Nan Wang ${ }^{1 \#}$, Fangqi Liu ${ }^{2 \#}$, Wenqi $\mathrm{Xi}^{1}$, Jinling Jiang ${ }^{1}$, Yun $\mathrm{Xu}^{2}$, Bingjie Guan ${ }^{1}$, Junwei $\mathrm{Wu}^{1}$, \\ Chenfei Zhou ${ }^{1}$, Min Shi ${ }^{1}$, Zhenggang Zhu ${ }^{1,3}$, Ye Xu ${ }^{2}$, Jing Liu ${ }^{1}$, Jun Zhang ${ }^{1}$ \\ ${ }^{1}$ Department of Oncology, Ruijin Hospital, Shanghai Jiao Tong University School of Medicine, Shanghai, China; ${ }^{2}$ Department of Colorectal \\ Surgery, Fudan University Shanghai Cancer Center, Shanghai, China; ${ }^{3}$ Shanghai Institute of Digestive Surgery, Ruijin Hospital, Shanghai Jiao Tong \\ University School of Medicine, Shanghai, China \\ Contributions: (I) Conception and design: Y Xu, J Liu, J Zhang; (II) Administrative support: J Zhang, Z Zhu, Y Xu; (III) Provision of study materials \\ or patients: F Liu, W Xi, J Jiang, J Wu, C Zhou, M Shi; (IV) Collection and assembly of data: N Wang, B Guan, Y Xu, J Liu; (V) Data analysis and \\ interpretation: J Liu, J Zhang, Y Xu; (VI) Manuscript writing: All authors; (VII) Final approval of manuscript: All authors. \\ "These authors contributed equally to this work. \\ Correspondence to: Jing Liu. Department of Oncology, Ruijin Hospital, Shanghai Jiao Tong University School of Medicine, No. 197 Ruijin Er Road, \\ Shanghai 200025, China. Email: 1j12255@rjh.com.cn; Ye Xu. Department of Colorectal Surgery, Fudan University Shanghai Cancer Center, \\ Dong'an Road, 270, Shanghai 200032, China. Email: yexu@shmu.edu.cn.
}

Background: Bone metastases (BM) from colorectal cancer (CRC) are often accompanied by extraosseous metastases, resulting in a dismal prognosis. The present study aimed to determine the risk factors for BM in metastatic CRC (mCRC) and the prognostic factors for CRC patients with BM.

Methods: The study was based on a training cohort of 214 mCRC patients (of which, 101 patients had $\mathrm{BM}$ ) from our center, and a validation cohort of 511 mCRC patients (of which, 173 patients had BM) from another institute. Risk and prognostic nomograms for BM were developed using univariate and multivariate analyses. The goodness of fit, discrimination, and calibration performance of the nomograms were assessed by $\mathrm{R}^{2}$, concordance statistics (C-statistics), and the calibration curve. The results were internally validated using bootstrap resampling in the training cohort, and externally validated in the validation cohort.

Results: The novel BM risk nomogram comprised seven variables [degree of tumor differentiation, N-stage, serum alkaline phosphatase (ALP), lactate dehydrogenase (LDH), carcinoembryonic antigen (CEA), liver metastasis, and lung metastasis]. It showed good performance, with an $\mathrm{R}^{2}$ of 0.447 and a C-statistic of 0.846 [95\% confidence interval (CI), 0.793 to 0.898 ] in the training cohort, and an $\mathrm{R}^{2}$ of 0.325 and a C-statistic of 0.792 (95\% CI, 0.750 to 0.834 ) in the validation cohort. The optimal cutoff value to identify individuals at low or high risk was $56 \%$ probability, with a sensitivity of $71.3 \%$ and a specificity of $89.4 \%$. The prognostic nomogram included five factors (tumor differentiation, number of extra-BM organs, number of BM lesions, ALP, and LDH), and had an $\mathrm{R}^{2}$ of 0.284 and a C-statistic of 0.723 (95\% CI, 0.657 to 0.789 ) in the training set. This nomogram was externally validated in the validation cohort, with an $\mathrm{R}^{2}$ of 0.182 and a C-statistic of 0.682 (95\% CI, 0.638 to 0.726 ).

Conclusions: The developed and validated risk and prognostic nomograms showed good performance for predicting the occurrence of BM in $\mathrm{mCRC}$ as well as the prognosis of CRC patients with BM. The risk nomogram can be used as a cost-effective preliminary screening tool prior to bone scanning.

Keywords: Colorectal cancer (CRC); bone metastases (BM); risk factors; prognosis; nomogram

Submitted Mar 22, 2021. Accepted for publication May 19, 2021.

doi: 10.21037/atm-21-2550

View this article at: http://dx.doi.org/10.21037/atm-21-2550 


\section{Introduction}

Colorectal cancer (CRC) is the third most common malignancy worldwide, and is responsible for nearly 2,000,000 new cases and 900,000 deaths every year (1). Distant metastases are the leading causes of CRC-related mortality, which occur in approximately $50 \%$ of patients after aggressive surgery. The most frequent metastatic organs are the liver and lungs, followed by lymph nodes, peritoneum, or brain (2-4).

Bone metastases (BM), often secondary from breast, prostate, and lung cancers, are not common in CRC patients $(5,6)$. The incidence of BM from CRC is 3-7\% in clinic but $10.7-23.7 \%$ in autopsy, indicating that a considerable number of occult BM are misdiagnosed during therapy $(7,8)$. Until now, the diagnosis of BM has been entirely dependent on imaging techniques, partially inclusive of bone scintigraphy (BS), magnetic resonance imaging (MRI), and positron emission tomography/ computed tomography (PET/CT). However, due to the low incidence of $\mathrm{BM}$ among CRC patients, high-cost imaging examination for $\mathrm{BM}$ is not recommended for routine follow-up until obvious symptoms of skeletal-related events (SREs) occur (9). SREs include (but are not limited to) disturbance of homeostasis, pathological fracture, and spinal cord compression, which could lead to a significantly poor quality of life and increased costs of nursing care (10). In addition, distant metastasis involving the skeletal system definitely predicts a remarkably worse prognosis than that confined to the liver or lungs in CRC partly due to a lack of effective treatment, as median overall survival (OS) after diagnosis of $\mathrm{BM}$ ranges from 5 to 21 months and the 1-year survival rate is only approximately $30 \%(7,11,12)$. To sum up, the main clinical characteristics of CRC patients with $\mathrm{BM}$ include low incidence, missed or delayed diagnosis, limited treatment methods, poor prognosis and poor quality of life, which make BM a big problem and challenge in clinical practice.

Several studies have identified the risk and prognostic factors associated with BM. The most well-established risk factors for BM are location of cancer, lymph node invasion and lung metastases (7). However, most studies also included patients with CRC who did not develop metastases (13-15), therefore the association found in the analysis most likely reflected the increased risk of all metastases. The incidence of BM is higher when only including metastatic CRC (mCRC) patients, accounting for nearly $10 \%$ (7), and $\mathrm{BM}$ is often complicated by extraosseous metastases, which tend to be overlooked by some arresting manifestations resulting from other organ metastases. We believe it is more clinically beneficial to identify people at a high risk for BM in mCRC. To date, there is no risk model has been developed for predicting BM only including mCRC patients. Thus, the purpose of this study was to establish and validate a clinical risk model to predict the occurrence of $\mathrm{BM}$ in $\mathrm{mCRC}$ and a prognostic model for CRC patients with BM. We present the following article in accordance with the TRIPOD reporting checklist (available at http:// dx.doi.org/10.21037/atm-21-2250).

\section{Methods}

\section{Patients and study design}

This retrospective study was conducted on a training cohort of 214 CRC patients with distant metastases treated in Ruijin Hospital Affiliated to Shanghai Jiao Tong University School of Medicine between January 2005 and May 2019. The inclusion criteria were as follows: (I) patients with histopathologically-confirmed colorectal adenocarcinoma through biopsy or surgery; (II) patients with metastatic disease; (III) patients with known status of bone metastasis; (IV) patients with no history of other malignancies; (V) patients with complete medical history and follow-up information. Patients who died of operative complications or non-neoplastic diseases were excluded. The selected patients were categorized into two groups: one group with BM (BM group, 101 cases), and the other group without BM from onset to death (NBM group, 113 cases).

From June 2006 to February 2018, an independent cohort of 511 CRC patients with distant metastases from Fudan University Shanghai Cancer Center was studied, using the same inclusion and exclusion criteria. There were 173 patients in the BM group and 318 patients in the NBM group. These patients formed the validation cohort of this study.

This study was conducted in accordance with the Declaration of Helsinki (as revised in 2013) and was approved by the Ruijin Hospital Ethics Committee of Shanghai Jiao Tong University School of Medicine (No.: 2020-318). The requirement for individual consent for this retrospective analysis was waived.

\section{Data collection}

Patients' demographics and tumor variables, including 
age at diagnosis of CRC, gender, metastatic sites, treatment for primary tumor, primary tumor site, degree of tumor differentiation, T-stage, $\mathrm{N}$-stage, and several serum biochemical indices, were collected via reviewing medical records or telephone follow-up. Pathological stage was not available in patients who did not undergo surgery, and clinical stage was recorded instead. Serum biochemical indices were recorded at first diagnosis of $\mathrm{BM}$ in the $\mathrm{BM}$ group and at first diagnosis of metastases in the NBM group. According to the standards developed by the clinical laboratory of Ruijin Hospital, serum biomarkers including alkaline phosphatase (ALP), lactate dehydrogenase (LDH), carcinoembryonic antigen (CEA), and carbohydrate antigen 19-9 (CA19-9) were determined, with upper normal limits of $126 \mathrm{IU} / \mathrm{L}, 192 \mathrm{IU} / \mathrm{L}, 5 \mathrm{ng} / \mathrm{mL}$, and $35 \mathrm{U} / \mathrm{mL}$, respectively. In the validation cohort, the upper normal limits were $125 \mathrm{U} / \mathrm{L}, 250 \mathrm{U} / \mathrm{L}, 5.2 \mathrm{ng} / \mathrm{mL}$, and $25.2 \mathrm{U} / \mathrm{mL}$, respectively.

In the BM group, we also collected some BM-related information, including treatment for BM (surgery, radiotherapy, chemotherapy, and bisphosphonate treatment), location of $\mathrm{BM}$ (pelvis, vertebrae, bones of the extremities, thoracic bones, or skull), number of BM lesions (based on the above five locations of BM), number of extra$\mathrm{BM}$ organs (number of metastatic organs other than BM), BM-free survival (BMFS, defined as the time from the diagnosis of CRC to bone metastasis), and OS (overall survival). The BM group was sub-grouped into 'synchronous BM', which was defined as BMFS of less than 1 month, and 'metachronous BM', which was defined as BMFS of more than 1 month.

The outcomes of the prediction models were overall survival (OS) and the occurrence of BM from onset to death. OS was defined as the time from the date of first diagnosis of BM to death or the date of the last known follow-up. The cutoff date for data analysis was the last follow-up on December 31, 2019 in the training cohort and on October 11, 2019 in the validation cohort. To ensure the blind assessment of the outcomes and predictors, the authors performed data entry and analysis independently from each other, and code names were used to replace each variable in the analysis.

\section{Diagnosis and Follow-up}

The clinical diagnosis of BM was identified using imaging examinations, including BS, MRI, CT, or PET/CT. Followup consisted of blood tests, as well as physical and imaging examinations (chest, abdomen, and pelvic CT or MRI scan with contrast) every 2 months; BM lesions were primarily observed by MRI.

\section{Statistical analysis}

Descriptive data were presented as medians with ranges (minimum to maximum) for continuous variables, and as frequencies and percentages for categorical variables. In the training cohort, univariate analyses were used to identify risk factors of BM by logistic regression models and prognostic factors in BM patients by Cox proportional hazard models. To obtain the optimal risk and prognostic models, a new multivariate logistic regression model and new multivariate Cox proportional hazard model were developed by entering a different set of variables, which were meaningful $(\mathrm{P}<0.05$ or clinically significant $)$ in the univariate analyses, one at a time. Two nomograms were formulated based on the results of the best prediction set. The optimal cutoff value was obtained through receiver operating characteristic (ROC) analysis to create risk groups. The goodness of fit of the nomograms was assessed by $\mathrm{R}^{2}$, and its discrimination was evaluated using the area under the curve (AUC) or concordance statistics (C-statistics). Calibration performance was assessed based on agreement between predicted and actual rates in the calibration curve. In the training cohort, the two models were internally validated by bootstrap resampling based on 1,000 resamples. External validation was performed in the validation cohort to analyze the same performance metrics, including $\mathrm{R}^{2}$, C-statistics, and calibration curve. SPSS version 24.0 (IBM Corp., Chicago, USA) and R version 3.6.1 (R Foundation for Statistical Computing, Vienna, Austria, http://www.r-project. org) were used for all statistical analyses. A two-sided $\mathrm{P}$ value $<0.05$ was considered statistically significant.

\section{Results}

\section{Patients' demographics and clinicopathologic characteristics}

In the training cohort, a total of 214 CRC patients with distant metastases were ultimately included in the final analysis, of whom, 101 (47.2\%) patients were diagnosed with BM. The median age at diagnosis of CRC was 59 years (range, 25-79 years) in the BM group, and 58 years (range, 24-79 years) in the NBM group, and the majority of patients were male $(62.4 \%$ in $\mathrm{BM}, 73.5 \%$ in NBM). 
Among the 113 patients without BM, the most common metastatic organ was the liver $(63.7 \%)$, followed by the lungs $(38.9 \%)$. Fifty-nine $(52.2 \%)$ patients were primary stage $\mathrm{IV}$, while the remaining patients were postoperative recurrent or metastasis. In the BM group, $25(24.8 \%)$ patients had synchronous metastasis and 76 (75.2\%) patients had metachronous metastasis. The average time from initial CRC diagnosis to detection of BM was 22.01 months (range, 0-123.6 months). Among whom 11 (10.9\%) patients were bone metastasis alone. The vertebrae were the most common metastatic site $(75,74.3 \%)$, followed by the pelvis $(50,49.5 \%)$. Only seven $(6.9 \%)$ patients underwent surgery for BM, including stabilization of pathological fractures, segmental resection of tumors, arthroplasty for replacing joints, and spinal decompression surgery. Most of the patients had non-surgical palliative treatment including chemotherapy, bisphosphonate, or radiotherapy.

In the validation cohort, $511 \mathrm{mCRC}$ patients from another medical center were enrolled in this study according to the same inclusion criteria, among whom, 173 (33.9\%) patients were in the BM group and 338 (66.1\%) patients were in the NBM group. The detailed demographics and clinicopathologic characteristics of patients in the training and validation cohorts are listed in Table 1. The differences in metastasis patterns between colon and rectal cancer in the training cohort are shown in Table S1. Among all groups, liver and lung were the most common metastatic sites. And colon cancer was more prone to peritoneal metastasis than rectal cancer. In BM group, we found that lung was the most common metastatic site in rectal cancer. The characteristics related to skeletal metastasis are shown in Table 2.

\section{Univariate analysis of risk factors for BM in the training cobort}

The results of the univariate analysis of risk factors for $\mathrm{BM}$ in the training cohort are listed in Table 3. We found that tumor differentiation degree of G3, N2 stage, and elevated serum ALP/LDH/CEA were risk factors for BM, and lung metastasis $(\mathrm{P}=0.09)$ was clinically significant. Liver metastasis was a protective factor for $\mathrm{BM}(\mathrm{P}<0.05)$. Among these, the serological indicators were included in the analysis as categorical variables according to the normal

Table 1 Demographics and clinicopathologic characteristics of CRC patients with and without BM

\begin{tabular}{|c|c|c|c|c|}
\hline Patients characteristics & \multicolumn{2}{|c|}{ Training cohort $(\mathrm{N}=214)$} & \multicolumn{2}{|c|}{ Validation cohort $(\mathrm{N}=511)$} \\
\hline \multicolumn{5}{|l|}{ Gender } \\
\hline Male & $63(62.4 \%)$ & $83(73.5 \%)$ & $113(65.3 \%)$ & $205(60.7 \%)$ \\
\hline Female & $38(37.6 \%)$ & $30(26.5 \%)$ & $60(34.7 \%)$ & $133(39.3 \%)$ \\
\hline \multicolumn{5}{|c|}{ Age at CRC diagnosed (yr) } \\
\hline Median [range] & 59 [25-79] & 58 [24-79] & 59 [29-90] & 56 [22-79] \\
\hline$\leq 60$ & $56(55.4 \%)$ & $66(58.4 \%)$ & $90(52.0 \%)$ & $217(64.2 \%)$ \\
\hline$>60$ & $45(44.6 \%)$ & $47(41.6 \%)$ & $83(48.0 \%)$ & $121(35.8 \%)$ \\
\hline \multicolumn{5}{|l|}{ Primary tumor site } \\
\hline \multicolumn{5}{|c|}{ Degree of tumor differentiation } \\
\hline $\mathrm{G} 1-2$ & $46(45.5 \%)$ & $70(61.9 \%)$ & $102(59.0 \%)$ & $225(66.6 \%)$ \\
\hline G3 & $55(54.5 \%)$ & $43(38.1 \%)$ & $71(41.0 \%)$ & $113(33.4 \%)$ \\
\hline
\end{tabular}

Table 1 (continued) 
Table 1 (continued)

\begin{tabular}{|c|c|c|c|c|}
\hline Patients characteristics & \multicolumn{2}{|c|}{ Training cohort $(\mathrm{N}=214)$} & \multicolumn{2}{|c|}{ Validation cohort $(\mathrm{N}=511)$} \\
\hline \multicolumn{5}{|l|}{ T-stage } \\
\hline T1-3 & $27(26.7 \%)$ & $30(26.5 \%)$ & $61(35.3 \%)$ & $61(18.0 \%)$ \\
\hline $\mathrm{T} 4$ & $74(73.3 \%)$ & $83(73.5 \%)$ & $112(64.7 \%)$ & 277 (82.0\%) \\
\hline NO & $16(15.8 \%)$ & $37(32.7 \%)$ & $31(17.9 \%)$ & $74(21.9 \%)$ \\
\hline N1 & $28(27.7 \%)$ & $47(41.6 \%)$ & $61(35.3 \%)$ & $146(43.2 \%)$ \\
\hline N2 & $57(56.4 \%)$ & $29(25.7 \%)$ & $81(46.8 \%)$ & $118(34.9 \%)$ \\
\hline \multicolumn{5}{|c|}{ Treatment for primary tumor } \\
\hline Radiotherapy & $16(15.8 \%)$ & $9(8.0 \%)$ & $44(25.4 \%)$ & $75(22.2 \%)$ \\
\hline \multicolumn{5}{|l|}{ ALP (IU/L) } \\
\hline Median (range) & $135(39-1,018)$ & $84(47-307)$ & $121(30-946)$ & $77.6(24.2-754.1)$ \\
\hline Normal & $42(41.6 \%)$ & $93(82.3 \%)$ & $92(53.2 \%)$ & $302(89.3 \%)$ \\
\hline High & $59(58.4 \%)$ & $20(17.7 \%)$ & $81(46.8 \%)$ & $36(10.7 \%)$ \\
\hline \multicolumn{5}{|l|}{ LDH (IU/L) } \\
\hline Median (range) & $220(68-4,863)$ & $166(90-6,496)$ & $222(103-2,748)$ & $169(93-2,111)$ \\
\hline Normal & $38(37.6 \%)$ & $83(73.5 \%)$ & $106(61.3 \%)$ & 285 (84.3\%) \\
\hline \multicolumn{5}{|l|}{ CA19-9 (U/mL) } \\
\hline Median (range) & $56.5(<0.8-2,627.1)$ & $56.5(<0.8->20,400)$ & $44.25(0.6-2,111)$ & $24.55(0.6-2,085)$ \\
\hline Normal & $43(42.6 \%)$ & $60(53.1 \%)$ & $71(41.0 \%)$ & 177 (52.4\%) \\
\hline High & $58(57.4 \%)$ & $53(46.9 \%)$ & $102(59.0 \%)$ & $161(47.6 \%)$ \\
\hline \multicolumn{5}{|c|}{ Metastatic sites (other than BM) } \\
\hline Liver & $48(47.5 \%)$ & $72(63.7 \%)$ & $79(45.7 \%)$ & $231(68.3 \%)$ \\
\hline Lung & $51(50.5 \%)$ & $44(38.9 \%)$ & $88(50.9 \%)$ & $94(27.8 \%)$ \\
\hline Peritoneum & $14(13.9 \%)$ & $17(15.0 \%)$ & $29(16.8 \%)$ & $50(14.8 \%)$ \\
\hline Distant lymph node & $42(41.6 \%)$ & $15(13.3 \%)$ & $39(22.5 \%)$ & $13(3.8 \%)$ \\
\hline Others & $25(24.8 \%)$ & $16(14.2 \%)$ & $47(27.2 \%)$ & $25(7.4 \%)$ \\
\hline
\end{tabular}

$\mathrm{BM}$, bone metastases; NBM, without bone metastases; CRC, colorectal cancer; ALP, alkaline phosphatase; LDH, lactate dehydrogenase; CEA, carcinoembryonic antigen; CA19-9, carbohydrate antigen 19-9. 
Table 2 Characteristics related to bone metastases

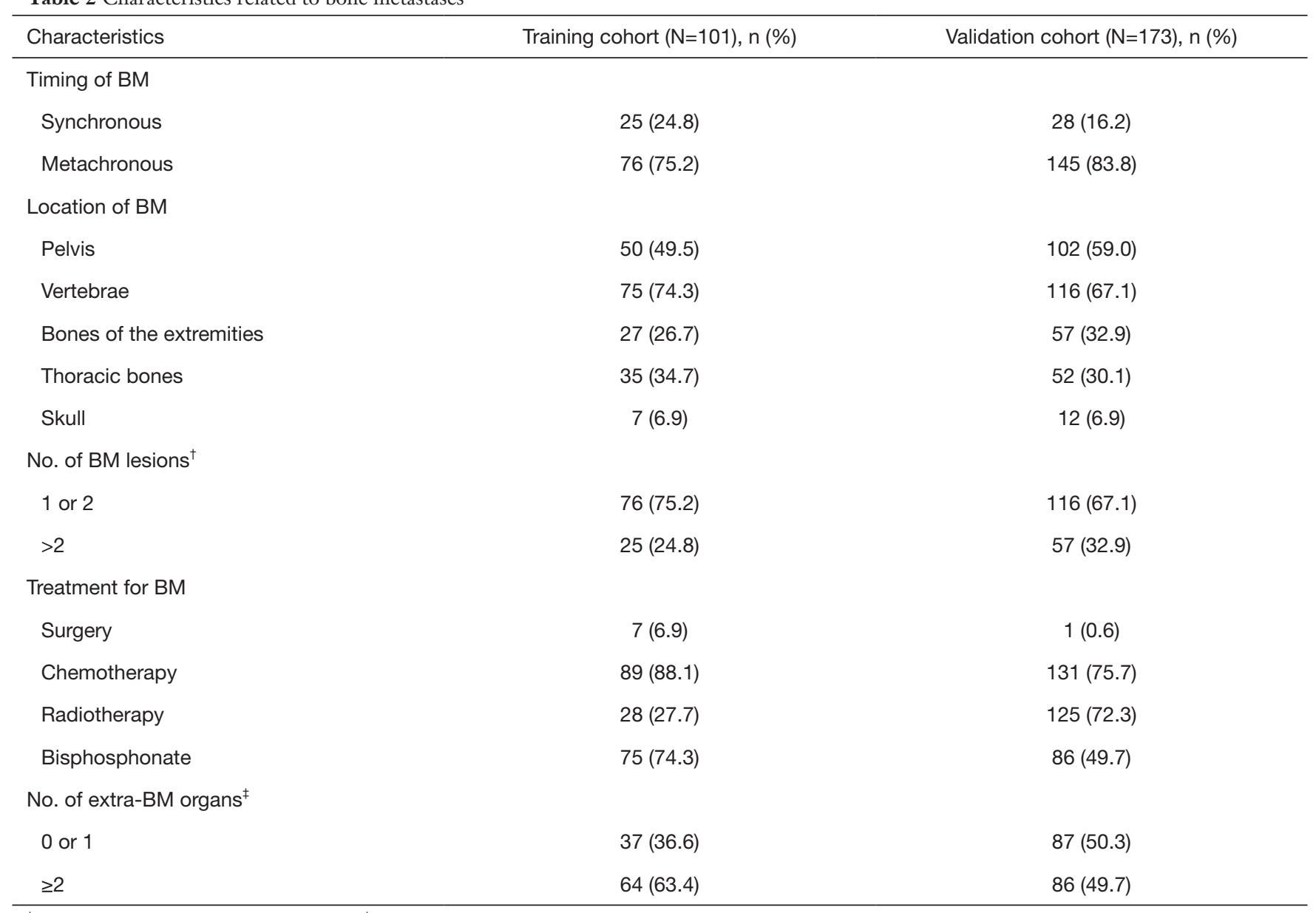

\footnotetext{
${ }^{\dagger}$, based on the five locations of $\mathrm{BM} ;{ }^{\ddagger}$, number of metastatic organs other than BM. BM, bone metastases.
}

value of clinical standards.

\section{Univariate analysis of prognostic factors in CRC patients with BM in the training cohort}

In the survival analysis, the median follow-up time was 26.5 months (range, 0.9-138.2 months). The median OS was 8.6 months (range, 0.5-49.9 months), and the 6-month, 1 -year, and 2-year OS rates were $63.4 \%, 32.7 \%$, and $8.9 \%$, respectively. The results of the univariate analysis are listed in Table 4. For prognostic factors of BM patients, the serological indicators were included in the analysis both as categorical and continuous variables. The results demonstrated that degree of tumor differentiation, number of extra-BM organs, number of BM lesions, ALP (categorical and continuous), and LDH/CEA/CA19-9 (categorical) were significantly meaningful for the prognosis of BM patients.

\section{Development of the risk nomogram for BM}

The risk nomogram that integrated the predictors $(\mathrm{P}<0.05$ or clinically significant) of $\mathrm{BM}$ in the univariate analysis is shown in Figure 1. The characteristics of the predictors in the multivariate logistic regression model are listed in Table $5(\mathrm{P}<0.001)$. Among them, $\mathrm{N}$-stage, serum ALP and $\mathrm{LDH}$, liver metastasis, and lung metastasis were identified as independent predictors in the model ( $\mathrm{P}$ value: 0.019 , $<0.001,0.017,<0.001$, and 0.014 respectively). The risk nomogram exhibited adequate goodness-of-fit, with an $\mathrm{R}^{2}$ of 0.447 , and showed good discrimination, with a C-statistic of 0.846 [ $95 \%$ confidence interval (CI), 0.793 to 0.898 ]. As the total points increased, the risk of $\mathrm{BM}$ also increased. When a $56 \%$ risk probability was applied as the optimal cutoff value through receiver operating characteristic (ROC) analysis to identify individuals at low or high risk, 
Table 3 Univariate analysis of risk factors for BM in the training cohort

\begin{tabular}{|c|c|c|}
\hline Variable & \multicolumn{2}{|c|}{ Univariate analysis } \\
\hline Sex (female vs. male) & $1.669(0.934-2.981)$ & 0.084 \\
\hline Age & $0.995(0.972-1.019)$ & 0.699 \\
\hline \multicolumn{3}{|l|}{ Primary tumor site } \\
\hline Right colon vs. rectum & $0.622(0.305-1.270)$ & 0.193 \\
\hline Degree of tumor differentiation (G3 vs. G1-2) & $1.946(1.128-3.358)$ & 0.017 \\
\hline T-stage (T4 vs. T1-3) & $0.991(0.540-1.818)$ & 0.976 \\
\hline \multicolumn{3}{|l|}{$\mathrm{N}$-stage } \\
\hline Liver metastasis (yes vs. no) & $0.516(0.298-0.891)$ & 0.018 \\
\hline Lung metastasis (yes vs. no) & $1.600(0.929-2.754)$ & 0.090 \\
\hline ALP (high vs. normal) & $6.532(3.498-12.197)$ & $<0.001$ \\
\hline LDH (high vs. normal) & $4.587(2.568-8.192)$ & $<0.001$ \\
\hline CEA (high vs. normal) & $3.983(1.902-8.341)$ & $<0.001$ \\
\hline CA19-9 (high vs. normal) & $1.527(0.889-2.621)$ & 0.125 \\
\hline
\end{tabular}

BM, bone metastases; CRC, colorectal cancer; ALP, alkaline phosphatase; LDH, lactate dehydrogenase; CEA, carcinoembryonic antigen; CA19-9, carbohydrate antigen 19-9; OR, odds ratio; Cl, confidence interval.

the sensitivity, specificity, and Youden index were $71.3 \%$, $89.4 \%$, and $60.7 \%$, respectively.

\section{Development of the prognostic nomogram for BM}

For the prognostic nomogram of BM patients, a different set of meaningful prognostic factors in the univariate analysis were entered to the multivariate Cox regression model to obtain an optimal prediction set. Among them, serum CEA and CA19-9 (categorical) were eliminated because their contribution to the model was insignificant (i.e., little difference between C-statistics with or without them). Serum ALP (continuous) was included as it provided more information than ALP (categorical). Finally, the prognostic nomogram comprised tumor differentiation, number of extra$\mathrm{BM}$ organs, number of BM lesions, serum ALP (continuous), and LDH (as shown in Figure 2). The characteristics of the predictors are listed in Table $5(\mathrm{P}<0.001)$. The model showed the good performance with an $\mathrm{R}^{2}$ of 0.284 and a C-statistic of 0.723 (95\% CI, 0.657 to 0.789 ).

\section{Comparison of predictive accuracy between the nomograms and single independent factor}

As shown in Table 5, the odds ratio and hazard ratio of ALP (high $v s$. normal) and number of extra-BM organs ( $\geq 2 v s .0$ or 1) for BM risk and survival were higher than the other factors, and they were significantly independent risk as well as prognostic factors. The predictive power between the two nomograms and these two factors was compared. The C-statistic for BM risk prediction by ALP (high vs. normal) was 0.704 , which was significantly lower than the C-statistic for $\mathrm{BM}$ risk prediction by the risk nomogram (0.846; $\mathrm{P}<0.001)$. Furthermore, the $\mathrm{C}$-statistic for OS prediction of BM patients by number of extra-BM organs ( $\geq 2$ vs. 0 or 1 ) was 0.646 , which was also lower than the $\mathrm{C}$-statistic by the prognostic nomogram $(0.723 ; \mathrm{P}=0.066)$.

\section{Internal and external validation of the two models}

The risk model was internally validated through bootstrap 
Table 4 Univariate analysis of prognostic factors in CRC patients with BM in the training cohort

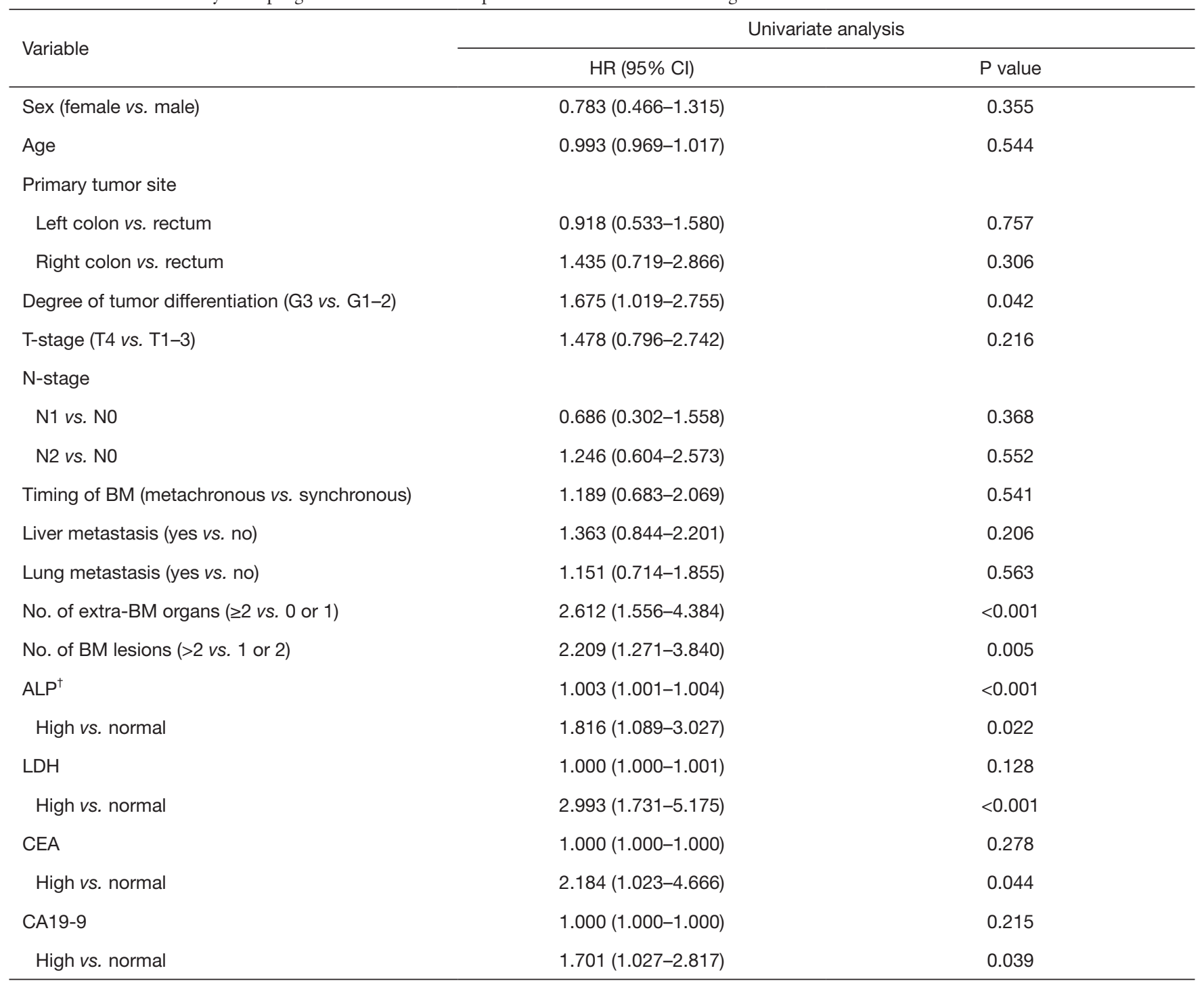

${ }^{\dagger}$, for continuous variable, same as LDH, CEA, CA19-9. BM, bone metastases; CRC, colorectal cancer; ALP, alkaline phosphatase; LDH, lactate dehydrogenase; CEA, carcinoembryonic antigen; CA19-9, carbohydrate antigen 19-9; HR, hazard ratio; Cl, confidence interval.

resampling $(\mathrm{B}=1,000)$, with a corrected $\mathrm{R}^{2}$ of 0.387 and a corrected C-statistic of 0.822 , which was similar to the original performance. External validation of the validation cohort from another hospital also showed great performance, with an $\mathrm{R}^{2}$ of 0.325 and a C-statistic of 0.792 (95\% CI, 0.75 to 0.834 ). Also, the model was well calibrated in both the training and validation cohorts, as shown in the calibration curve (method = 'boot', $\mathrm{B}=1,000)$ (Figure $3 A, B)$.

For the prognostic model, the corrected $\mathrm{R}^{2}$ was 0.224 and the corrected C-statistic was 0.705 in the training cohort (method $=$ 'boot', $\mathrm{B}=1,000)$. In the validation cohort, the median follow-up time was 35.2 months (range, 0.8-175.0 months). The median OS was 10.9 months (range, 0.8-134.7 months), and the 6-month, 1-year, and 2-year OS rates were $74.6 \%, 45.1 \%$, and $21.4 \%$, respectively. The performance metrics in the validation cohort (external validation) showed good generalization ability, with an $\mathrm{R}^{2}$ of 0.182 and a C-statistic of 0.682 (95\% CI, 0.638 to 0.726). The calibration plot for the probability of survival at 1 year after diagnosis of BM showed an optimal agreement between the nomogram prediction and actual observation both in the training and validation cohorts (method = 'boot', 


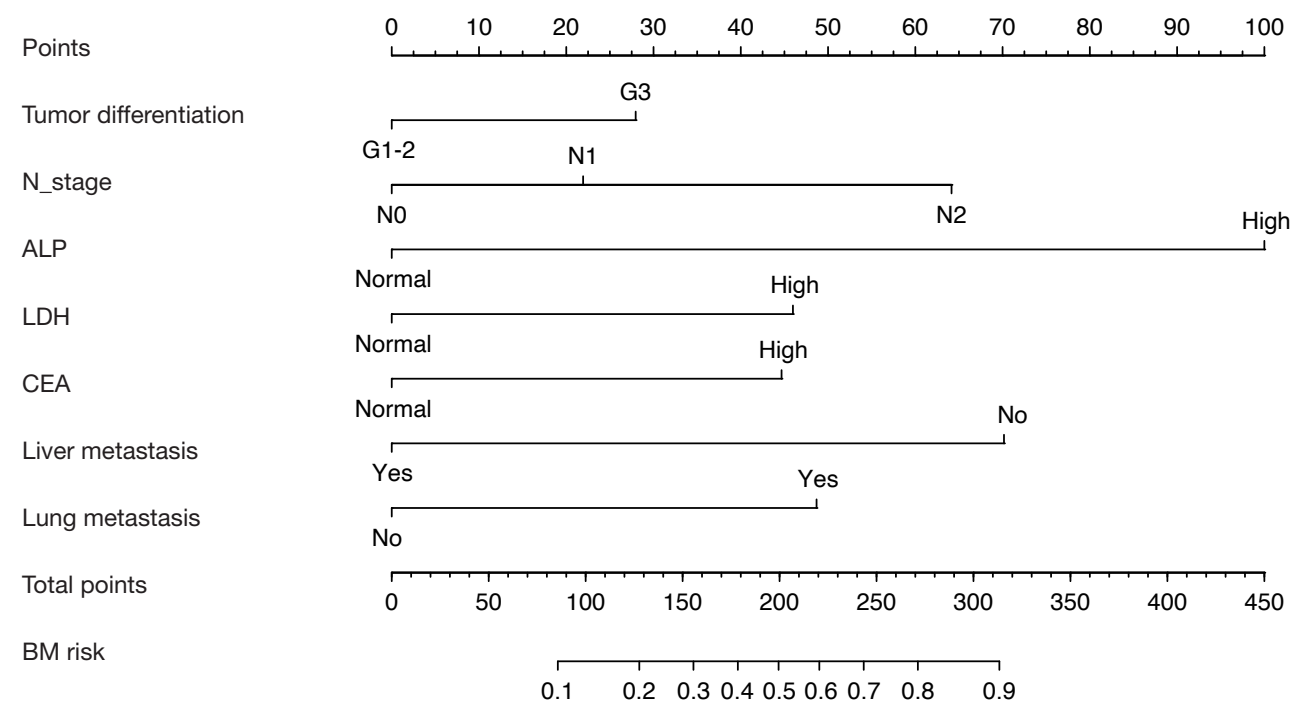

Figure 1 Nomogram model for predicting individual risk of BM in mCRC (risk nomogram). BM, bone metastases; mCRC, metastatic colorectal cancer; N-stage, regional lymph node metastasis of primary tumor; ALP, alkaline phosphatase; LDH, lactate dehydrogenase; CEA, carcinoembryonic antigen.

Table 5 Predictors in the risk and prognostic nomograms for BM using multivariate logistic/Cox regression

\begin{tabular}{|c|c|c|}
\hline & Adjusted OR/HR (95\% Cl) & $P$ value \\
\hline Differentiation (G3 vs. G1-2) & $1.715(0.834-3.529)$ & 0.143 \\
\hline $\mathrm{N}$-stage & & 0.019 \\
\hline N1 vs. N0 & $1.527(0.625-3.731)$ & 0.353 \\
\hline ALP (high vs. normal) & $6.89(3.119-5.219)$ & $<0.001$ \\
\hline LDH (high vs. normal) & $2.429(1.174-5.026)$ & 0.017 \\
\hline CEA (high vs. normal) & $2.368(0.941-5.959)$ & 0.067 \\
\hline Liver metastasis (yes vs. no) & $0.258(0.122-0.546)$ & $<0.001$ \\
\hline Differentiation (G3 vs. G1-2) & $1.340(0.777-2.313)$ & 0.293 \\
\hline No. of extra-BM organs ( $\geq 2$ vs. 0 or 1 ) & $2.357(1.355-4.098)$ & 0.002 \\
\hline No. of BM lesions (>2 vs. 1 or 2 ) & $1.452(0.786-2.684)$ & 0.233 \\
\hline LDH (high vs. normal) & $1.961(1.060-3.632)$ & 0.032 \\
\hline $\mathrm{ALP}^{\dagger}$ & $1.191(0.964-1.472)$ & 0.105 \\
\hline
\end{tabular}

${ }^{\dagger}$, continuous variable. BM, bone metastases; ALP, alkaline phosphatase; LDH, lactate dehydrogenase; CEA, carcinoembryonic antigen; $\mathrm{HR}$, hazard ratio; OR, odds ratio; $\mathrm{Cl}$, confidence interval. 


Points
No. of extra-BM organs
No. of BM-lesions
Tumor differentiation
LDH
ALP (IU/L)
Total points
1-year survival
2-year survival
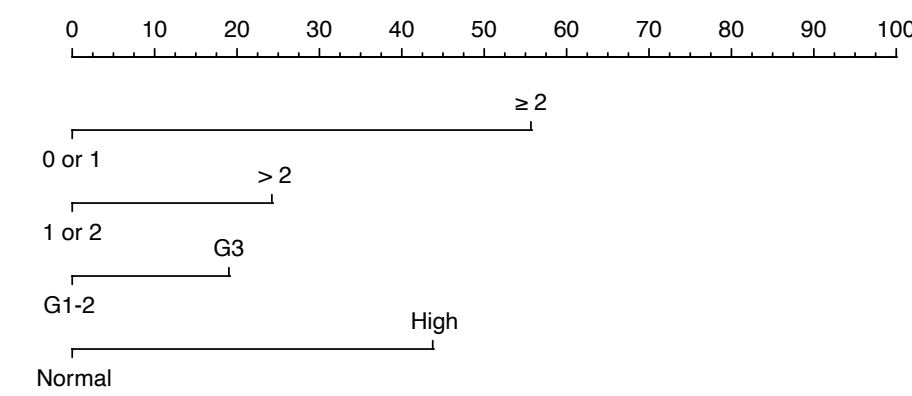

Normal

2-year survival

\begin{tabular}{|c|c|c|c|c|c|c|c|c|c|c|c|c|}
\hline 0 & 100 & 200 & 300 & 400 & 50 & 0 & 600 & 700 & 800 & 900 & 1000 & 1100 \\
\hline 0 & 20 & 40 & 60 & 80 & 100 & 120 & 140 & 160 & 180 & 200 & 220 & 240 \\
\hline 0.8 & & 0.7 & 0.6 & 0.5 & 0.4 & 0.3 & 0.2 & 0.1 & 0.05 & & & \\
\hline 0.6 & 0.5 & 0.4 & 0.3 & 0.2 & 0.1 & 0.05 & & & & & & \\
\hline
\end{tabular}

Figure 2 Nomogram model for predicting the 1- or 2-year survival probability in CRC patients with BM (prognostic nomogram). BM, bone metastases; CRC, colorectal cancer; ALP, alkaline phosphatase; LDH, lactate dehydrogenase.

$\mathrm{B}=1,000, \mathrm{~m}=25 / 40)($ Figure $3 C, D)$.

\section{Discussion}

In the present study, a novel risk nomogram comprising the degree of tumor differentiation, N-stage, liver metastasis, lung metastasis, as well as serum ALP, LDH, and CEA, and a new prognostic nomogram comprising tumor differentiation, number of extra-BM organs, number of BM lesions, ALP (continuous), and LDH, were developed and validated to provide more accurate risk and prognosis prediction for BM. The two models had good performance both in the training and validation cohorts, supported by the C-statistics ( 0.846 and 0.792 for the risk model; 0.723 and 0.682 for the prognostic model, respectively) and calibration curves. In addition, individuals were divided into low- and high-risk groups based on the optimal cutoff value (56\% risk probability) in the risk nomogram. We recommended that patients at high risk should be further examined by either BS, MRI, or PET-CT to evaluate the BM status during follow-up.

In recent decades, survival rates of mCRC have been improved due to multidisciplinary discussion, individualized and comprehensive therapy, in particular, molecular targeted therapy and biological immunotherapy. Molecular biomarkers like RAS/BRAF/PIK3CA [the key driver genes mutations of CRC (16)], microsatellite instability (MSI) status and etc., guiding targeted and immuno-therapy, have been successfully applied in clinical practice. However, the treatment of mCRC still has great challenges, more than $85 \%$ of mCRC has no specific driver genes (17). Especially for CRC patients with BM, at present, effective treatments for $B M$ are quite limited, include bisphosphonate to alleviate SREs, systemic chemotherapy to control tumor, radiotherapy or surgery to relieve symptoms, and medications to relieve pain (18). Unfortunately, there are no active chemotherapy drugs target BM lesions, whether local treatment can prolong survival is still unclear (7). The prerequisite is early detection of $\mathrm{BM}$, before the lesions spread.

In clinical practice, CAP (chest/abdominal/pelvic) CT scans are routinely recommended for mCRC patients during follow-up; however, when and which patients need to perform bone scans remains uncertain, causing delayed or missed diagnosis. Due to delayed diagnosis and absence of effective therapies, BM patients achieved a severely short survival time with only 8.6 months (in the training cohort) and 10.9 months (in the validation cohort) in our study. Thus, a thorough understanding of the risk and prognostic predictors for $\mathrm{BM}$ is important to identify individuals at high risk who may need further accurate examination, particularly for patients who already harbor other metastases, and to better improve the judgment of prognosis of BM patients.

A good screening tool needs to be low cost, provide clinical utility, and exhibit relatively high accuracy. We 


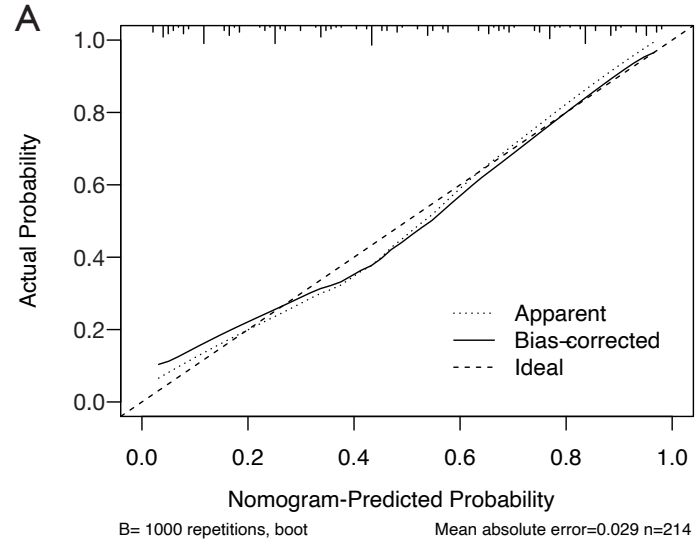

C

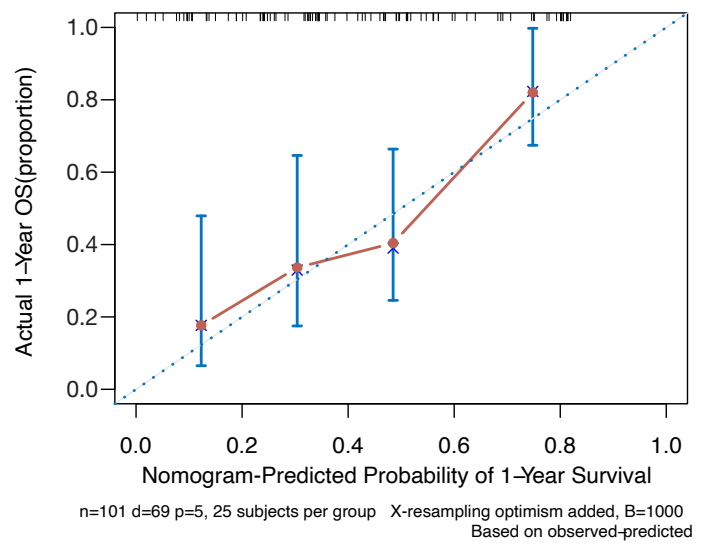

B

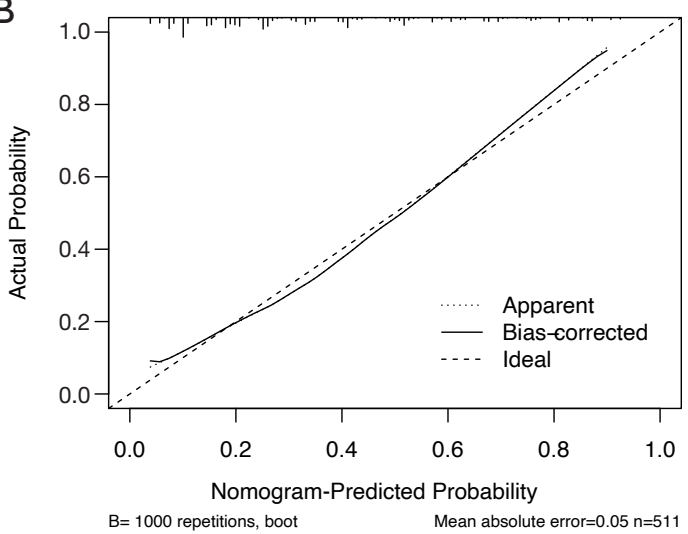

$\mathrm{D}$

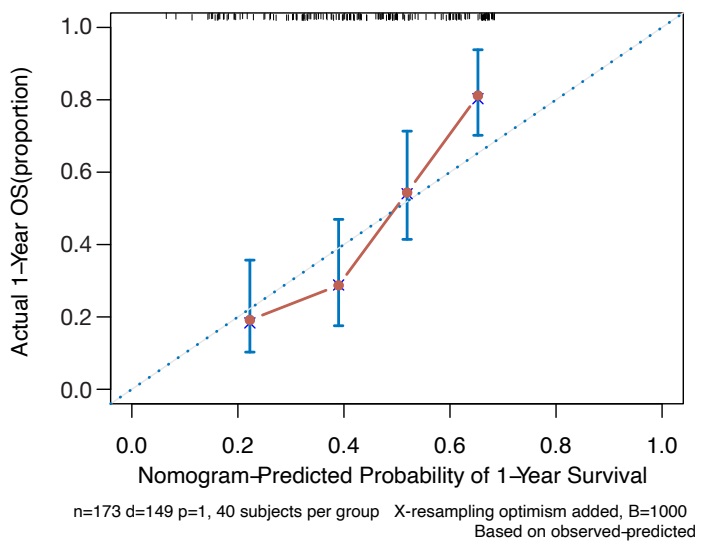

Figure 3 Calibration curves of the risk and prognostic nomogram. Calibration curves of the risk nomogram for predicting individual risk of $\mathrm{BM}$ in the training cohort (A) and the validation cohort (B). Calibration curves of the prognostic nomogram for predicting the 1-year survival probability of CRC patients with BM in the training cohort (C) and the validation cohort (D). BM, bone metastases; CRC, colorectal cancer.

developed the first risk nomogram as a prescreening tool to predict the risk probability of BM in mCRC patients. Our nomogram included only seven variables, and all of them were routinely examined items during the followup. Consistent with previous studies $(9,13,14), \mathrm{G} 3$ tumor differentiation and lymph node involvement of the primary tumor were included in our risk model, which may demonstrate that a high degree of malignancy of the primary tumor is associated with the risk of BM. A positive CEA result may indicate the presence of residual tumor after radical surgery or a higher tumor burden, leading to a greater likelihood of bone infiltration and metastasis. In addition, several studies have demonstrated that lung metastasis is a potential risk factor for developing BM $(9,13,15)$. For liver metastasis, the results were inconclusive and controversial, possibly due to ethnic differences and different research objects $(9,19)$. In our study, liver metastasis was found to be a protective factor for BM in the mCRC patient cohort. A possible explanation for this phenomenon may be that the intrinsic biological characteristics of the tumor might lead to the heterogeneity and mutual exclusivity of metastatic organs. For example, Cristescu et al. (20) found that in gastric cancer, there were a higher percentage of subjects with peritoneal seeding in the microsatellite stable (MSS)/epithelial-mesenchymal transition (EMT) subtype; but in this subtype, few patients developed liver metastasis.

Our study also found that elevated levels of ALP and $\mathrm{LDH}$ significantly increased the risk of $\mathrm{BM}$ and resulted in a poor prognosis. ALP is viewed as an indirect reflection of 
bone turnover and has been widely applied for the diagnosis and evaluation of bone diseases $(21,22)$. Several studies have reported that increased ALP levels are an independent risk factor for BM in many tumor types, including CRC, leading to high sensitivity and positive predictive values for BM, as well as poor clinical outcomes (23-27). When cancer cells infiltrate into the bones, they can stimulate osteoclasts to induce bone destruction and activate osteoblasts, resulting in increased serum ALP (28). Therefore, the elevation of ALP, to some extent, indicates the formation of metastatic bone lesions. Serum ALP also increases with the tumor load of BM, leading to a worse prognosis. Furthermore, bone ALP (bALP) is preferable for predicting BM owing to its higher specificity. Lim et al. (29) found that bALP might be a surrogate marker of bone metastasis in gastric cancer patients, with a sensitivity of $76.7 \%$ and a specificity of $59.4 \%$.

The essence of elevated ALP is the change of bone microenvironment, which plays a critical role in the formation of BM. Cells and cytokines in bone marrow and bone matrix together constitute the bone microenvironment (30). It remains not fully understood how CRC cells interact with bone microenvironment. Cancer cells can disrupt the delicate balance between the functional cells in the bone microenvironment (i.e., osteoblasts and osteoclasts) to create structural defects and change the bone "soil" leading to a more favorable environment for metastasis and growth of cancer cells. In this process, chemokines also play a key role. Different chemokines like CXCL12, CXCR4, CCL3, etc. participate in the chemoattraction of CRC cells to bone tissue and promote cancer cell metastasis (31).

In addition, $\mathrm{LDH}$ is another cytoplasmic enzyme expressed throughout tissues in the whole body. Elevated LDH levels seem to imply an underlying tumor burden or an aggressive phenotype. Under intracellular hypoxia (resulting from cancerous excessive metabolism), LDH predominantly facilitates the tumor-specific Warburg effect, producing adequate energy via aerobic glycolysis and secreting lactate as a waste product, which likely dominates the tricarboxylic acid cycle (TCA) cycle as a carbon source after re-uptake by cancer cells $(32,33)$. Kang et al. (34) demonstrated that higher serum LDH was correlated with the occurrence of SREs, and several studies also found that LDH served as a predictive factor for BM development and prognosis of BM patients in some tumor types $(33,35,36)$. In our study, serum LDH contributed to both the risk and prognostic models.
In our prognostic nomogram, besides ALP and LDH, 'number of BM lesions' was also a prognosis factor. In recent years, multiple BMs have come to the foreground due to their strong correlation with poor prognosis in BM-prone cancers. Multiple BMs, especially metastases involving both axial and extremital skeletons, were identified as strong factors related to poorer survival in patients with advanced breast cancer (37). Moreover, a previous study focused on the close association between multiple BM and increased incidence of SREs (34). As an illustration, multiple BMs, especially those involving weight-bearing skeletons, were inclined toward spinal cord compression, and bone pain was commonly magnified by multiple osteolytic lesions, which continuously undermined the quality of life of patients. It is worth noting that 'number of BM lesions', which was a prognostic factor in our study, was classified as $>2$ and 1 or 2, based on the five locations of BM (pelvis, vertebrae, bones of the extremities, thoracic bones, or skull). Furthermore, the majority of patients $(89.1 \%$ in training cohort) in the BM group were complicated by extraosseous metastases in our study, often in the lung and/or liver, which is consistent with previous reports. Liu et al. (27) found that CRC patients with BM alone seemed to form a distinct group with a better prognosis. Our study showed that ' $\geq 2$ extraBM organs' was a strong independent factor correlated with prognosis, similar to a recent study from Japan (11). An explanation for this may be that excessive organ metastases with a high tumor burden tend to lead to multiple organ failures, and thus influence survival. Clinicians should pay more attention to these patients during follow-up.

There were several potential limitations in the present study that should be noted. Firstly, although the models were internally and externally validated, both the training and validation cohorts involved retrospective data, and there is a lack of prospective cohorts to verify the accuracy and stability. Secondly, some novel bone resorption markers, such as tartrate-resistant acid phosphatase, which is more specific for bone metabolism, and some known prognostic factors, like the occurrence of SREs, were not available in this study due to insufficient data. What's more, the data of genes for most patients in this study were not available, partly because genetic testing was not a routine program in early years. Recent data suggest that gene mutation may related to specific metastatic organs in CRC. For example, some studies have found that BRAF mutation is associated with peritoneal spread $(38,39)$. However, there are few studies on genes of specific bone metastasis, and it is worthy of further investigation. In future research, we will add 
prognostic-related genes and conduct in-depth research on the functions and pathways of genes of specific bone metastasis in CRC patients.

\section{Conclusions}

In conclusion, the risk and prognostic nomograms developed and validated in the present study showed a good performance in identifying individuals at higher risk of BM among mCRC patients and BM patients with worse outcomes. The risk nomogram can be used as a convenient and cost-effective preliminary screening tool prior to bone scanning. In the future, large prospective studies are needed to validate the efficacy and feasibility of our nomograms.

\section{Acknowledgments}

Funding: This work was supported by the National Science Foundation of China [81802319]; the Shanghai RisingStar Program [20QA1406200]; the Shanghai Municipal Commission of Health and Family Planning [20184Y004]; the Guangci Distinguished Young Scholars Training Program [GCQN-2018-A06]; and the Shanghai Science and Technology Committee Foundation (No. 18140903702).

\section{Footnote}

Reporting Checklist: The authors have completed the TRIPOD reporting checklist. Available at http://dx.doi. org/10.21037/atm-21-2250

Data Sharing Statement: Available at http://dx.doi. org/10.21037/atm-21-2250

Conflicts of Interest: All authors have completed the ICMJE uniform disclosure form (available at http://dx.doi. org/10.21037/atm-21-2250). The authors have no conflicts of interest to declare.

Ethical Statement: The authors are accountable for all aspects of the work in ensuring that questions related to the accuracy or integrity of any part of the work are appropriately investigated and resolved. This study was conducted in accordance with the Declaration of Helsinki (as revised in 2013) and was approved by the Ruijin Hospital Ethics Committee of Shanghai Jiao Tong University School of Medicine (No.: 2020-318). The requirement for individual consent for this retrospective analysis was waived.
Open Access Statement: This is an Open Access article distributed in accordance with the Creative Commons Attribution-NonCommercial-NoDerivs 4.0 International License (CC BY-NC-ND 4.0), which permits the noncommercial replication and distribution of the article with the strict proviso that no changes or edits are made and the original work is properly cited (including links to both the formal publication through the relevant DOI and the license). See: https://creativecommons.org/licenses/by-nc-nd/4.0/.

\section{References}

1. Bray F, Ferlay J, Soerjomataram I, et al. Global cancer statistics 2018: GLOBOCAN estimates of incidence and mortality worldwide for 36 cancers in 185 countries. CA Cancer J Clin 2018;68:394-424.

2. Kim HK, Cho JH, Lee HY, et al. Pulmonary metastasectomy for colorectal cancer: how many nodules, how many times? World J Gastroenterol 2014;20:6133-45.

3. Weiss L, Grundmann E, Torhorst J, et al. Haematogenous metastatic patterns in colonic carcinoma: an analysis of 1541 necropsies. J Pathol 1986;150:195-203.

4. August DA, Ottow RT, Sugarbaker PH. Clinical perspective of human colorectal cancer metastasis. Cancer and Metastasis Reviews 1984;3:303-24.

5. Brown JE, Cook RJ, Major P, et al. Bone turnover markers as predictors of skeletal complications in prostate cancer, lung cancer, and other solid tumors. J Natl Cancer Inst 2005;97:59-69.

6. Vatandoust S, Price TJ, Karapetis CS. Colorectal cancer: Metastases to a single organ. World J Gastroenterol 2015;21:11767-76.

7. Christensen TD, Jensen SG, Larsen FO, et al. Systematic review: Incidence, risk factors, survival and treatment of bone metastases from colorectal cancer. J Bone Oncol 2018;13:97-105.

8. Katoh M, Unakami M, Hara M, et al. Bone metastasis from colorectal cancer in autopsy cases. J Gastroenterol 1995;30:615-8.

9. Guan X, Ma CX, Quan JC, et al. A clinical model to predict the risk of synchronous bone metastasis in newly diagnosed colorectal cancer: a population-based study. BMC Cancer 2019;19:704.

10. Baek SJ, Hur H, Min BS, et al. The Characteristics of Bone Metastasis in Patients with Colorectal Cancer: A Long-Term Report from a Single Institution. World J Surg 2016;40:982-6.

11. Kawamura H, Yamaguchi T, Yano Y, et al. 
Characteristics and Prognostic Factors of Bone Metastasis in Patients With Colorectal Cancer. Dis Colon Rectum 2018;61:673-8.

12. Khattak MA, Martin HL, Beeke C, et al. Survival differences in patients with metastatic colorectal cancer and with single site metastatic disease at initial presentation: results from South Australian clinical registry for advanced colorectal cancer. Clin Colorectal Cancer 2012;11:247-54.

13. Li A, Kasmann L, Rades D, et al. A Scoring System to Predict the Development of Bone Metastasis After Radical Resection of Colorectal Cancer. Anticancer Res 2017;37:5169-72.

14. Sun C, Deng Y, Zhou H, et al. Risk factors for the development of metachronous bone metastasis in colorectal cancer patients after curative resection. Int J Surg 2015;21:145-9.

15. Zhenghong, Zihua Z, Guoweijian, et al. Retrospective study of predictors of bone metastasis in colorectal cancer patients. J Bone Oncol 2017;9:25-8.

16. Huang D, Sun W, Zhou Y, et al. Mutations of key driver genes in colorectal cancer progression and metastasis. Cancer Metastasis Rev 2018;37:173-87.

17. De Falco V, Napolitano S, Roselló S, et al. How we treat metastatic colorectal cancer. ESMO Open 2020;4:e000813.

18. Krempien R, Niethammer A, Harms W, et al. Bisphosphonates and bone metastases: current status and future directions. Expert Rev Anticancer Ther 2005;5:295-305.

19. Sundermeyer ML, Meropol NJ, Rogatko A, et al. Changing patterns of bone and brain metastases in patients with colorectal cancer. Clin Colorectal Cancer 2005;5:108-13.

20. Cristescu R, Lee J, Nebozhyn M, et al. Molecular analysis of gastric cancer identifies subtypes associated with distinct clinical outcomes. Nat Med 2015;21:449-56.

21. Farley JR, Baylink DJ. Skeletal alkaline phosphatase activity as a bone formation index in vitro.

Metabolism1986;35:563-71.

22. Romagnoli E, Minisola G, Carnevale V, et al. Assessment of serum total and bone alkaline phosphatase measurement in clinical practice. Clin Chem Lab Med 1998;36:163-8.

23. Zhou Y, Yu QF, Peng AF, et al. The risk factors of bone metastases in patients with lung cancer. Sci Rep 2017;7:8970.

24. Li AA, Cao ZY, Liu JM, et al. The risk factors for bone metastases in patients with colorectal cancer. Medicine (Baltimore) 2018;97:e12694.

25. Chen WZ, Shen JF, Zhou Y, et al. Clinical characteristics and risk factors for developing bone metastases in patients with breast cancer. Sci Rep 2017;7:11325.

26. Matsuyama H, Shimabukuro T, Hara I, et al. Combination of hemoglobin, alkaline phosphatase, and age predicts optimal docetaxel regimen for patients with castrationresistant prostate cancer. Int J Clin Oncol 2014;19:946-54.

27. Liu F, Zhao J, Xie J, et al. Prognostic risk factors in patients with bone metastasis from colorectal cancer. Tumour Biol 2016. [Epub ahead of print]. doi: 10.1007/ s13277-016-5465-4.

28. Mountzios G, Ramfidis V, Terpos E, et al. Prognostic significance of bone markers in patients with lung cancer metastatic to the skeleton: a review of published data. Clin Lung Cancer 2011;12:341-9.

29. Lim SM, Kim YN, Park KH, et al. Bone alkaline phosphatase as a surrogate marker of bone metastasis in gastric cancer patients. BMC Cancer 2016;16:385.

30. Fornetti J, Welm AL, Stewart SA. Understanding the Bone in Cancer Metastasis. J Bone Miner Res 2018;33:2099-113.

31. Gigante I, Tutino V, De Nunzio V, et al. Colorectal Cancer and Bone Tissue: Fantastic Relations and Where to Find Them. Cancers 2020;12:2029.

32. Faubert B, Li KY, Cai L, et al. Lactate Metabolism in Human Lung Tumors. Cell 2017;171:358-71.e9.

33. Armstrong AJ, Eisenberger MA, Halabi S, et al. Biomarkers in the management and treatment of men with metastatic castration-resistant prostate cancer. Eur Urol 2012;61:549-59.

34. Kang EJ, Lee SY, Kim HJ, et al. Prognostic Factors and Skeletal-Related Events in Patients with Small Cell Lung Cancer with Bone Metastases at the Time of Diagnosis. Oncology 2016;90:103-11.

35. Katakami N, Kunikane H, Takeda K, et al. Prospective study on the incidence of bone metastasis (BM) and skeletal-related events (SREs) in patients (pts) with stage IIIB and IV lung cancer-CSP-HOR 13. J Thorac Oncol 2014;9:231-8.

36. Conen K, Hagmann R, Hess V, et al. Incidence and predictors of Bone Metastases (BM) and Skeletal-Related Events (SREs) in Small Cell Lung Cancer (SCLC): A Swiss patient cohort. J Cancer 2016;7:2110-6.

37. Parkes A, Warneke CL, Clifton K, et al. Prognostic Factors in Patients with Metastatic Breast Cancer with Bone-Only Metastases. Oncologist 2018;23:1282-8.

38. Yaeger R, Cercek A, Chou JF, et al. BRAF mutation predicts for poor outcomes after metastasectomy in patients with metastatic colorectal cancer. Cancer 
2014; 120:2316-24.

39. Tran B, Kopetz S, Tie J, et al. Impact of BRAF mutation and microsatellite instability on the pattern of metastatic spread and prognosis in metastatic colorectal cancer.

Cite this article as: Wang N, Liu F, Xi W, Jiang J, Xu Y, Guan B, Wu J, Zhou C, Shi M, Zhu Z, Xu Y, Liu J, Zhang J. Development and validation of risk and prognostic nomograms for bone metastases in Chinese advanced colorectal cancer patients. Ann Transl Med 2021;9(10):875. doi: 10.21037/atm21-2250
Cancer 2011;117:4623-32.

(English Language Editor: A. Kassem) 
Supplementary

Table S1 Differences in metastasis patterns between colon cancer and rectal cancer in the training cohort

\begin{tabular}{|c|c|c|c|c|}
\hline \multirow{2}{*}{$\begin{array}{l}\text { Metastatic sites (other } \\
\text { than BM) }\end{array}$} & \multicolumn{2}{|c|}{ BM Group (N=101) } & \multicolumn{2}{|c|}{ NBM Group (N=113) } \\
\hline & Rectal cancer $(n=56)$ & Colon cancer $(n=45)$ & Rectal cancer $(n=61)$ & Colon cancer $(n=52)$ \\
\hline Liver & $22(39.3 \%)$ & $26(57.8 \%)$ & $39(63.9 \%)$ & $33(63.5 \%)$ \\
\hline Lung & $33(58.9 \%)$ & $18(40 \%)$ & $25(41 \%)$ & 19 (36.5\%) \\
\hline Distant lymph node & $23(41.1 \%)$ & $19(42.2 \%)$ & $6(9.8 \%)$ & $9(17.3 \%)$ \\
\hline Others & $11(19.6 \%)$ & $14(31.1 \%)$ & $7(11.5 \%)$ & $9(17.3 \%)$ \\
\hline
\end{tabular}

BM, bone metastases; NBM, without bone metastases. 